\title{
ESTADO Y MIGRACIÓN: ORGANIZACIONES TRANSNACIONALES Y DESARROLLO LOCAL EN SINALOA, MÉXICO ${ }^{1}$
}

\section{STATE AND IMMIGRATION: TRANSNATIONAL ORGANIZATIONS AND LOCAL DEVELOPMENT IN SINALOA, MEXICO}

\author{
Renato Pintor Sandoval* \\ Ernesto Sánchez Sánchez**
}

RESUMEN

El presente artículo analiza las formas de interacción y gestión de los clubes de migrantes sinaloenses y su impacto socioeconómico en sus lugares de origen. En primera instancia se analiza el desempeño del Club San José, que apoyado por la Fraternidad Sinaloense en California, tiene un impacto social de manera transnacional a través de prácticas y vínculos comunitarios. En el segundo apartado, se analiza la Fraternidad Elotense en California, que amparados bajo las iniciativas de los presidentes municipales en turno y bajo el esquema del programa 3x1, desarrollan inversiones de infraestructura local en beneficio de la localidad de origen, sobrepasando el principio fundacional del grupo de migrantes organizados que reside en mantener viva las actividades comunitarias de forma transnacional.

PALABRAS CLAVE: MIGRANTES * ORGANIZACIÓN * COMUNIDAD * ESTADO * POLÍTICAS PÚBLICAS

\section{ABSTRACT}

This article analyzes the behavior of Sinaloa's migrant clubs and their social and economic impact in their places of origin. Firstly, the actions of the San José Club are analyzed, which, supported by the Sinaloan Fraternity in California, makes it possible to have a social impact in a transnational manner through practices and community links. Secondly, the Elotense Fraternity in California is analyzed, which under the initiatives of the municipal presidents in turn and under the scheme of the 3x1 program, allow

$1 \quad$ El presente trabajo fue realizado bajo el apoyo del PROFAPI 2015/222 y PROFAPI 2015/267. Proyectos financiados por la Universidad Autónoma de Sinaloa, México. sansan.ernesto@gmail.com 
the development of local infrastructure investments in favor of the locality of origin, surpassing the group of organized migrants' founding principle, which is to keep community activities alive transnationally.

KEYWORDS: MIGRANTS * ORGANIZATION * COMMUNITY * STATE * PUBLIC POLICIES

\section{INTRODUCCIÓN}

Desde inicios del siglo xxI, se han desarrollado de manera exponencial investigaciones de los distintos aportes que realizan las organizaciones de migrantes en sus lugares de origen, en las cuales se analizan los efectos sociales, económicos y culturales que estas desarrollan; así como, las formas iniciales de la organización, recaudación, gestión, cabildeo y supervisión. El dinamismo histórico de la migración mexicana ha permitido la creacion $y$ la consolidadción de organizaciones, fraternidades $y$ clubes de migrantes, que a lo largo de 15 años, en conjunción con los diferentes niveles de gobierno, han intervenido en el desarrollo regional de los lugares de origen de los migrantes.

En 2017, se registraron más de 500 clubes de migrantes mexicanos que radican en Estados Unidos. La unión de cinco o más clubes generó la conformación de federaciones de migrantes, que funciona de manera autónoma y sin fines de lucro. En 2002, se creó el Programa $3 x 1$ que buscaba apoyar las iniciativas ciudadanas a partir de recursos de migrantes y de la federación, Estados y municipios. Este programa del Gobierno surgió a cargo de la Secretaría de Desarrollo Social (SEDESol) que apoya las iniciativas de los migrantes organizados para gestionar y realizar proyectos que busquen el desarrollo de sus localidades de origen.

Los proyectos van desde infraestructura social, servicios comunitarios, educativos y proyectos productivos, entre estos, construcción y rehabilitación de proyectos de redes de agua, saneamiento y potabilización; construcción, ampliación y rehabilitación de pavimentaciones, caminos y carreteras; becas académicas y apoyos para el aprendizaje y compra de útiles escolares y uniformes; alimentación y servicios de capacitación empresarial para proyectos productivos.
Lo anterior ha beneficiado cerca de 14 millones de mexicanos (SEDESOL, 2017). Estas inversiones sociales responden en parte al desarrollo histórico de la migración mexicana a Estados Unidos, por otra parte, son una necesidad ante la baja recaudación y partida presupuestal que se le otorgan a los municipios en México.

En este sentido, es importante analizar el impacto socioeconómico, más allá de la trascendencia que se deriva de sus inversiones. En otras investigaciones se enfatizó el peso decisivo por la cantidad de remesas que envían los migrantes, como una forma de dependencia de estos recursos en las comunidades de origen (Sánchez, García y Pintor, 2015). En este sentido, se muestran las diferentes formas operativas de la organización, gestión y agencia que llevan estas organizaciones. Para ello se analiza como referencia a dos clubes oriundos del Estado de Sinaloa. Se trata, en primera instancia, de hacer una caracterización de la maduración de los clubes, así como de los nuevos instrumentos que se han incorporado en las negociaciones $y$ los impedimentos que los aquejan una vez que sobrepasan una etapa a otra. Por esta razón, no es fortuito que se discuta si la inversión migrante puede transformar la estructura económica de sus lugares de destino.

En este artículo se sostiene que la dinámica de estos clubes y fraternidades es heterogénea; por un lado, son decisivos los liderazgos comunitarios como lo muestra el Club San José (Agua Verde, El Rosario) pionero en Sinaloa. Este club está suscrito en la Fraternidad Sinaloense en California $y$ ha dejado de participar paulatinamente en el programa $3 \times 1$; mientras que el otro, en la Fraternidad Elotense en California, es decisiva la participación del gobierno local como coparticipe de los programas bajo el esquema federal del $3 \times 1$. 


\section{CLUBES Y FRATERNIDADES MIGRANTES: EMPODERAMIENTO Y RECONOCIMIENTO}

Los clubes, fraternidades $y$ asociaciones migratorias mexicanas tienen un crecimiento considerable en las dos últimas décadas del siglo xx. El desempeño de estas organizaciones permite, através de sus gestiones, vincular a los migrantes con el desarrollo de sus comunidades y complementar su desarrollo, ya sea de manera autónoma, o bien, integrando a los gobiernos en sus tres niveles (federal, estatal y municipal), el sector privado o social (Hernández, 2007; Sánchez, García y Pintor, 2015).

La difusión del fenómeno organizacional de migrantes, no solo en Estados de tradición migratoria sino en los de reciente migración, es trascendental para la gestión e implementación en los programas gubernamentales en los 3 niveles. Actualmente, existen dos estrategias metodológicas para el abordaje de esta problemática: 1) sus obras o inversiones sociales y 2) la naturaleza de su organización. En cuanto a la primera, Goldring (2002, p.57) considera cuatro formas distintas de utilización de remesas colectivas:

$\diamond \quad$ Obras de pequeña infraestructura básica y de comunicación (caminos, puentes, agua potable, drenaje, tratamiento de aguas negras, pozos, electrificación, casetas telefónicas).

$\diamond \quad$ Infraestructura y capitalización de servicios públicos o de bienestar, es decir, proyectos relacionados a la educación, salud, $y$ seguro social (escuelas, computadoras, clínicas, ambulancias, despensas, asilos de ancianos).

$\diamond \quad$ Obras de recreación y estatus (canchas deportivas, lienzos).

$\diamond \quad$ Otras obras comunitarias o de recreación urbana (salones de usos múltiples, "casinos”, plazas, bancas, fachadas, arcos).

Asimismo, señala que los clubes por su simbolismo llevan acabo complejas y diferenciadas formas de representación en sus lugares de origen (Goldring, 2005), por ejemplo, donación de juguetes y materiales escolares, financiamiento a grupos artísticos, becas, construcción de asilos de ancianos, construcción de centros para la juventud y remodelación de carreteras, puentes, iglesias, escuelas o centros de salud.

No obstante, las dificultades que enfrentan las organizaciones de migrantes $y$ sus proyectos sociales han de concretarse en el impacto social en la comunidad de origen. Esto es precisamente uno de los objetivos primordiales de los clubes de migrantes, porque "tratan de no ser excluidos", buscando alcanzar una "integración social" y que en la distancia continuan manifestando normas, tradiciones, sociabilidades, apego social e involucramiento que logran mediante la construcción y la extensión de una organización filial.

El crecimiento de estas organizaciones es cada vez más grande; en el 2002, existían más de 400 asociaciones de migrantes en 18 Estados (Pintor, 2015). En el 2014, con la ayuda del Instituto de los Mexicanos en el Exterior (IME) y de manera autónoma, se registraran más de 1100 clubes en todo Estados Unidos, de los cuales, 300 eran de la región sur de California (tabla 1). Sin embargo, para el año 2015, SEDESOL señalaba que existían alrededor de 1800 asociaciones.

Para el caso de los clubes tradicionales y con autonomía en las inversiones, Moctezuma (2008) analiza que "este proceso sólo es posible con el patrón del migrante establecido, el cual no sólo se diferencia del patrón circular migratorio, $y$ también por el hecho de haberse transformado de migración individual a familiar" (p. 97) y de participar grupalmente. Esta perspectiva sirve fundamentalmente para comprender que el hilo conductor del desempeño organizacional no recae en la inversión, sino en la naturaleza de la organización migrante ${ }^{2}$. Esto hace diferente a la dinámica de los clubes de reciente creación auspiciada por la inversión pública que suelen manejarse bajo la agenda municipal, donde su aporte consiste en dar al

\footnotetext{
2 Para Moctezuma (2011) se ha hecho un juicio negativo a las organizaciones de migrantes con base a señalamiento políticos, pero se soslaya sus prácticas de carácter transnacional, así pues, los clubes de migrantes se reconocen en su naturaleza misma y en la transición de las redes sociales hacia el desarrollo de las comunidades filiales.
} 
migrante un espacio de participación mediante la obra pública.

En el debate comparativo sobre la dinámica de los clubes migratorios se señala, por parte de García (2005) y González (2006), la grandeza de estas organizaciones de migrantes por la cantidad de inversiones sociales y por las remesas colectivas que promueven y que inciden en el desarrollo local de las comunidades de origen. Lo anterior debido a la insistencia en cómo aprovechar los recursos del migrante en las zonas de expulsión. Este debate se centró primeramente en las remesas familiares, posteriormente, se sitúo en las remesas colectivas.

TABLA 1

CLUBES DE MIGRANTES MEXICANOS EN LOS ESTADOS UNIDOS POR LUGAR DE ORIGEN (PERIODOS 1998-2003 Y 2009-2011)

\begin{tabular}{l|cccc}
\hline \multicolumn{1}{c|}{ ESTADO } & 1998 & 2003 & 2009 & 2011 \\
\hline Jalisco & 74 & 100 & 31 & 93 \\
Michoacán & 19 & 51 & 55 & 22 \\
Zacatecas & 113 & 126 & 12 & 50 \\
Guanajuato & 40 & 48 & 307 & 12 \\
D.F. & 3 & 6 & 6 & 16 \\
Sinaloa & 12 & 17 & 5 & 75 \\
Durango & 19 & 20 & 54 & 10 \\
Nayarit & 22 & 27 & 5 & 64 \\
Guerrero & 23 & 51 & 15 & 61 \\
Puebla & 12 & 34 & 38 & 16 \\
Estado de México & 6 & 11 & 14 & 74 \\
Oaxaca & 22 & 36 & 74 & 134 \\
Hidalgo & 4 & 11 & 101 & 13 \\
Aguascalientes & 3 & 1 & 8 & 676 \\
Otros & 69 & 84 & 667 & 1842 \\
\hline Total & 441 & 623 & 1392 & \\
\hline
\end{tabular}

Fuente: Tomado de Escala Rabadán (2014) en base a los datos de González Zepeda (2012, p. 54) y SRE (2013). Los datos que ofrece la SRE son los mismos para el 2014 (año en que ya no apareció el registro en la página oficial de la Secretaría) por lo que se recomienda utilizar esta fuente con cierta cautela, ya que se trata de datos sobrerepresentados.

Los analisis de las remesas y las formas de actuar de los clubes migrantes han generado una importante discusión, no solo en el impacto sino en el abordaje metodológico del proceso migratorio. Así pues, a nivel teóricometodológico, la comprensión de la migración internacional implica una reformulación, ya no sujeta a las definiciones clásicas de migrante y de su status migratorio, sino a redefiniciones propias de "ser migrante". Las investigaciones de Smith (2006), Moctezuma (2011) o Goldring (2002 y 2005) han abordado el fenómeno migratorio de mexicanos hacia Estados Unidos con especial interés en las relaciones, prácticas $y$ negociaciones que guardan los individuos con sus lugares de origen detectando cualidades $y$ elementos simbólicos que antes no se incluían en su análisis.

Dado la complejidad, masividad e importancia socioeconómica que ha dejado la migración mexicana a Estados Unidos se han creado nuevas redes migratorias, pero siempre sustentadas en redes sociales históricas. Barnes (1954) define la red social como "Cada persona se relaciona con un cierto número de individuos, algunos de los cuales están en contacto 
directo entre sí y otros no"(p. 36). Para Rouse (1992), la red social migratoria es "el conjunto de lazos interpersonales que conectan a los migrantes en las áreas de origen $y$ destino, mediante lazos de parentesco, amistad y del compartir un origen común" (p.221).

En esta tesitura, se asocia a los "migrantes recientes", a sus estructuras de parentesco y de amistad, pues cada nuevo migrante crea un conjunto de lazos sociales. Los migrantes están inevitablemente ligados a los no-migrantes (familia y comunidad que no migra) y los segundos hacen uso de las obligaciones implícitas en las relaciones de parentesco y amistad para tener acceso al empleo y a la asistencia social en el lugar de destino.

Con base a Fitzgerald (2014), este proceso social es multifacético. Los acercamientos actuales del Estado mexicano tratan de administrar, regular y vigilar a casi dos de cada diez mexicanos que han dejado el país, creando un sentido de pertenecía común pero en la distancia, poniendo en marcha una gran cantidad de instrumentos, instituciones, programas o mecanismos de acercamiento (Escala, 2014). El desarrollo y consolidación de las redes sociales han permitido que se originen, primero, los grupos de oriundos llamados clubes de migrantes y segundo, las llamadas asociaciones de clubes de migrantes.

En el primero, los clubes o "comunidades filiales", los migrantes se reconocen $y$ se adhieren como parte de una sociedad. De acuerdo a datos de la Fraternidad Sinaloense en California, existen alrededor de 500000 sinaloenses en la Unión Americana, tanto en Estados como Utah, Nueva York, Illinois, Arizona, y Texas, pero principalmente en California. Este último contiene casi el $75 \%$ de la población sinaloense en Estados Unidos. La migración sinaloense a ese Estado no es reciente, sino que ha recibido constantes oleadas de migración a lo largo de los últimos 50 años. Su comportamiento migratorio permite que sea el punto de llegada, principalmente a las regiones de Los Ángeles, San Diego, el área de la Bahía de San Francisco y recientemente, hay una concentración en Arizona, Utah, Washington, Nevada y Carolina del Sur (Pintor, 2015).

\section{EL CLUB SAN JOSÉ}

En México han aumentado el número de clubes $y$ federaciones. La conformación de estas organizaciones se ha presentado bajo ásperas relaciones que tejen con sus diferentes gobiernos. Algunos autores las consideran como "optimistas" (postura que tienen algunas organizaciones migrantes para que en su conformación solucionen problemas de la comunidad sin trabas burocráticas) y se prevé son difíciles de concretar (conjunto de procedimientos administrativos-burocráticos que intervienen en la implementación de una política pública); entre estos, Valenzuela y Lanly (2004) y Padilla (2000) consideran que cuando se elaboran proyectos conjuntos, en ocasiones, la capacidad monetaria de los clubes "ha sido superior a las aportaciones que realizan los distintos gobiernos". Otro problema es la politización con la que se realizaban algunas obras sociales, por lo que los habitantes de los lugares de origen hacen un reconocimiento diferenciado de las obras que han sido "construidas por los migrantes" frente a las "construidas por el gobierno". Lo que es un hecho es el acercamiento del club con las autoridades políticas en cuanto al diseño de políticas públicas (Valenzuela, 2007).

Con respeto a California, espacio con un dinamismo e historicidad extraordinaria en el mapa migratorio mexicano, cuenta con la mayor cantidad de presencia de clubes y organizaciones de migrantes. Una de estas es la Fraternidad Sinaloense en California (FSC) fundada en 1991, y su misión es organizar a 14 clubes de oriundos californianos registrados hasta 2009. Las actividades que realizan son bailes de coronación de reinas y la suscripción de convenios con sus respectivas autoridades estatales, comprometiéndose así a secundar el trabajo social que llevan a cabo los clubes en las comunidades de origen (Revista de la Fraternidad Sinaloense, 1998 y 1998a; Pintor y García, 2008) (cuadro 1). 
CUADRO 1

CLUBES SINALOENSES INSCRITOS EN LA FRATERNIDAD SINALOENSE

Y SUS PRINCIPALES OBRAS SOCIALES $(2000)^{3}$

\begin{tabular}{|c|c|c|c|c|c|}
\hline $\begin{array}{l}\text { NOMBRE DEL } \\
\text { CLUB }\end{array}$ & $\begin{array}{c}\text { AÑO DE } \\
\text { FUNDACIÓN }\end{array}$ & PRESIDENTE & $\begin{array}{l}\text { FREC. DE } \\
\text { REUNIÓN }\end{array}$ & $\begin{array}{l}\text { PROYECTOS } \\
\text { PRINCIPALES }\end{array}$ & $\begin{array}{l}\text { MIEMBROS } \\
\text { ACTIVOS }\end{array}$ \\
\hline San Ignacio & 1993 & Adriana Noriega & No disponible & No disponible & No disponible \\
\hline Guasave & 1992 & Ramón Cárdenas & Cada 2 meses & $\begin{array}{c}\text { Apoyo } \\
\text { comunitario y } \\
\text { entrega de carro } \\
\text { de bomberos }\end{array}$ & 25 \\
\hline Rosario & 1985 & Octavio Solorza & Cada mes & $\begin{array}{l}\text { Rec. del asilo } \\
\text { y apoyos a la } \\
\text { comunidad }\end{array}$ & 20 \\
\hline Escuinapa & 1991 & Lourdes Arroyo & Cada mes & Jardín de niños & 25 \\
\hline Agua Verde & 1981 & Javier Benítez & Cada mes & $\begin{array}{l}\text { Construcción de } \\
\text { iglesia y biblioteca }\end{array}$ & 25 \\
\hline El Roble & 1992 & José Ibarra & Cada 2 meses & $\begin{array}{c}\text { Apoyos } \\
\text { comunitarios }\end{array}$ & 6 \\
\hline Los Pericos & 1993 & Leticia Camacho & Cada mes & No disponible & No disponible \\
\hline Mazatlán & 1993 & Octavio Barrón & Cada mes & $\begin{array}{l}\text { Desayunos } \\
\text { escolares, sistema } \\
\text { de alumbrado } \\
\text { en colonias } \\
\text { necesitadas }\end{array}$ & 50 \\
\hline Ahome & 1993 & José Zarate & Cada mes & $\begin{array}{l}\text { Envío de } 50 \text { sillas } \\
\text { de ruedas }\end{array}$ & 14 \\
\hline El Fuerte & 1993 & $\begin{array}{l}\text { Ma. del Refugio } \\
\text { Reyna }\end{array}$ & Cada mes & Carro de bomberos & 14 \\
\hline Culiacán & 1993 & Antonio Malacón & Cada mes & $\begin{array}{l}\text { Carro de bomberos } \\
\text { y remodelación de } \\
\text { la plazuela }\end{array}$ & 25 \\
\hline
\end{tabular}

Fuente: Revista de la Fraternidad Sinaloense, Los Ángeles, julio de 1998.

$3 \quad$ El Club Cubiri, el Club Caiteme, el Club Costa Rica y el Club Rosa Morada adquirieron su adscripción a la Fraternidad en el lapso de 2008-2009 y sus inicios para su formación fueron apoyados por el IME. 
Desde su establecimiento, auspiciado por el entonces Cónsul José Ángel Pescador Osuna y por Humberto Gálvez, ambos oriundos de Sinaloa, la FSC ha tenido como objetivo representar a la comunidad migrante ante las autoridades, tanto en México como en California. En palabras de José Ángel Barajas, la Fraternidad se visualiza como:

Una fuerte organización no lucrativa que cuenta con oficina $y$ centro de negocios, establecida en varias regiones de California; con una imagen sólida frente a la comunidad, con un desarrollo económico estable, así como con un incremento continuo en la adhesión de nuevas comunidades de sinaloenses en California, lo cual ha permitido forjar nuevos líderes (http://fraternidadsinaloense.com).

Para lograr objetivos de acercamiento e incidencia en la comunidad recibe el apoyo de SEDESOL por medio del programa $3 \times 1$, a través de proyectos de interés comunitario como carreteras, pavimentación de calles, electrificación, remodelación de iglesias y escuelas (Revista de la Fraternidad Sinaloense, 2008). Los diferentes líderes de las comunidades y el aval de la Fraternidad permiten nuevas estrategias para mejores proyectos que busquen el desarrollo económico de Sinaloa. Uno de los proyectos que realizó en 2008, fue un evento musical para ayudar al Asilo de Ancianos de Mazatlán, patrocinado por el grupo musical, cuyos integrantes radican en Phoenix, Arizona. En este evento se recaudó la cantidad de $\$ 2500$ dólares, que fueron donados y procesados bajo el programa $3 \times 1$, por lo que se haría llegar entonces la cantidad total de 10 mil dólares al asilo de ancianos en Mazatlán (Pintor y García, 2008). De igual forma, se firmaron proyectos denominados "Rescate de la Sierra", que tienen como objetivo principal que las comunidades de la sierra puedan contar con plantas de luz y agua, así como de piso de cemento, remodelación de caminos rurales y servicio médico a través de brigadas. Otro proyecto fue la recaudación de fondos destinados para el proyecto de la Universidad Polítecnica del Valle del Évora en Sinaloa, concretada en 2011 (Revista de la Fraternidad Sinaloense, 2008).

Con base a lo anterior, uno de los clubes sobresalientes es el Club de migrantes San José, cuyo nombre obedece al Santo Patrono de Agua Verde, localizado en el Rosario, Sinaloa. La socialización por el arraigo del migrante comenzó por la familia de Javier Benítez, reconocido porque sus padres fueron maestros de la escuela primaria de la localidad. Él es empresario en el sector de servicios, expandiéndose mediante una empresa de limpieza y escalando al liderazgo de una organización religiosa cristiana local en Los Angeles, California: All Saints (Pintor, 2015).

A finales de la década de los 70 en Agua Verde, el líder del Club tuvo contacto con el párroco de la iglesia local, y se acordó hacer una colecta para ayudar a construir un nuevo templo. La necesidad de reconstruir el templo del Santo Patrono del lugar se expandió paulatinamente entre los aguaverdenses emigrados en Los Ángeles, $y$ lograron reunirse para buscar la manera de hacer llegar la ayuda financiera. La gestión duró 3 años en cristalizarse, pero ya demostraba la existencia de los miembros a organizarse autónomamente sin la intervención del Estado. En marzo de 1982, durante la fiesta del santo patrón, inauguraron la fachada del templo y el atrio parroquial. Esta fue la acción fundacional del club de migrantes de Agua Verde y con esta acción comunitaria se obtuvo el reconocimiento social que dio a las personas colaboradoras la oportunidad de recuperar la membresía comunitaria que se había diluido por su condición de migrante.

Diversas son las enseñanzas del caso de Agua Verde, entre estas se encuentra la existencia de un proyecto social colectivo, que se impulsa a través de varios proyectos menores en los que se involucran las personas migrantes y otros agentes locales. Además, se observa que durante su inicio y en gran parte de su desarrollo social, sus inversiones sociales no contemplaron la aportación del gobierno, por el contrario, los aportes económicos los hicieron la población migrante en su totalidad. En este caso, Moctezuma (2011) señala que "se trata de estructuras sociales simples e informales que 
eran invisibles para el Gobierno Mexicano, pero que ya expresan el desarrollo de una membresía transnacional desde abajo" (p. 69).

También hay que destacar la experiencia, el poder alcanzado y el estatus social que las distingue como un club que logra sus objetivos, factores que la identifican como una instancia participativa y organizada. En los grupos formales, el estatus suele determinar la posición en la estructura, mientras que en los informales se basan en aspectos que parecen relevantes al grupo (Morán, 2002).

Este aprendizaje social, a través de la gestión e implementación de programas de intervención social, que deben experimentar los clubes de migrantes, los hace proclives al aplazamiento de las obras con un proceso lento; sin embargo, lo esencial es reconocer quién está interesado, qué funciones desempeñará, cuál es la situación de los migrantes para participar, ya que como señalan Gibson, Ivancevich y Donnelly (1994), las remesas colectivas e individuales pueden tardarse o nunca llegar, pero a pesar de ello, en este campo social se genera un espacio donde se recrean prácticas transnacionales.

Esto se observa con el Club All Saints. Las obras sociales necesitaron de un determinante extra para que se pudiera socializar, principalmente por la escasa participación o desinterés de miembros de la comunidad de origen $y$ esto contradijo su esencia transnacional. Ante ello, la siguiente misión del Club San José fue la biblioteca pública de la comunidad, donde la adquisición del acervo de 1000 libros aproximadamente $y$ las negociaciones emprendidas fue el resultado de varios años de colectas y actividades de la organización de migrantes aguaverdenses. Teresa Benítez, maestra de la escuela, señaló que uno de los miembros del club donó su casa para que se instalara la biblioteca. La maestra Benitez ha participado como intermediaria de algunos de los programas que realiza el club, señalando que los habitantes presumen que ya cuentan con una biblioteca (Pintor, 2015).

Más allá de las obras emprendidas por el Club San José, el caso de Agua Verde ejemplifica que "el sentimiento de pertenencia es la sensación o percepción de ser parte de un determinado grupo social, mediante el cual se toma distancia de otro" (Enríquez, 2000, pp.42), de los no participativos. En este sentido de pertenencia, los aguaverdenses desde la distancia marcan ya un alejamiento-acercamiento con el "otro". La persona migrante convive y recrea su vida misma a través de la participación en actividades que la involucren con su espacio, que a su vez es dicotómico con las prácticas que realiza.

Esto lleva a una reinterpretación de la identidad individual de los aguaverdenses emigrados y su adhesión a la comunidad. Esta puede estar determinada por el papel socializador al transmitirles la memoria histórica del grupo, la cosmovisión del mundo y también por un nuevo marco de relaciones sociales donde se dinamiza el mundo del trabajo y las nuevas redes de amistades que los conectan con otros grupos sociales. En este sentido, la constitución de la identidad individual es un proceso dinámico que está articulado con la identidad social del grupo de pertenencia (Giménez, 2010). Es decir, hay un sistema formado por migrantes que no solo se redefine por su condición identitaria, sino que se fortalece al incorporar una simbología novedosa. Aquí se manifiestan diferencias con otros migrantes y se crean identidades propias en las que se establecen "conexiones intersistémicas" (Molina, 2014, p. 28).

Entre los aportes que presenta el estudio de los clubes de migrantes por localidad de origen, se menciona la constante transformación del proceso transnacional, donde a la par de sus negociaciones, se van sumando nuevos agentes de cambio, disputas de poder y lazos que se refuerzan o se reestructuran. Sin embargo, no basta solo con el conocimiento, la composición y la historia de las organizaciones migrantes para detectar la nuevas formas de relaciones en estas comunidades. También replantearse que estas formas de organización del migrante supervisan y negocian su participación local, municipal y regional. Desarrollan una labor de aprendizaje político y social al ser gestores de proyectos de coinversión con sus distintos gobiernos, $y$ que en su recorrido sufren fracturas $y$ adhesiones, tanto en el ámbito interno como externo. 


\section{LA FRATERNIDAD ELOTENSE EN CALIFORNIA} (FEC)

Para Alba (2010), el inicio del nuevo siglo se caracterizó porque distintos gobiernos llevaron la dimensión migratoria y sus impactos económicos a políticas económicas a través de programas, esto es lo que se denomina la migratización de las políticas de desarrollo. De este modo, el gobierno asume de manera estratégica las bondades de las remesas colectivas, principalmente las familiares. Sin embargo, un punto a destacar de las remesas colectivas y la organización migrante es la escasa transferencia relativa y transparencia de recursos ${ }^{4}$. En este sentido, primeramente se hace referencia a los envíos de dinero vía electrónica y que se modifican ya que la cantidad está en función de la tasa de cambio dólar-peso y de los cargos e impuestos que se aplican por el envío y que termina por reducir la cantidad original, $y$ por el otro lado, la falta de transparencia en la rendición de cuentas de los recursos destinado a obras comunitarias.

Solo una mínima parte del total de las remesas familiares son transferibles por organizaciones de migrantes que envían dinero a sus comunidades para apoyar, no solo a la familia, sino a la construcción de infraestructura pública $y$ el desarrollo de proyectos sociales. Por su parte, el IME señala que "la creación y propagación de tantos clubes de migrantes por lugar de origen se debe, sin duda, a la ardua promoción del Instituto de los Mexicanos en el Exterior" (Zabin y Escala, 2002, p.101), postulando con esto la participación del Estado para agrupar a sus ciudadanos y ciudadanas más allá de las fronteras. Informalmente, también funcionan otro tipo de organizaciones como las deportivas que realizan los consulados o asociaciones religiosas del lugar de destino, las cuales son un ejemplo de los procesos de formación de las comunidades transnacionales que cultivan sus identidades fuera de sus lugares de origen (Alarcón, 2004).

$4 \quad$ En sus estudios sobre este tipo de recursos, Torres (2001) comenta que la organización, las formas $y$ el empuje que tienen estas organizaciones son recursos que bien pueden ser de alta calidad.
El caso de las personas migrantes del municipio de Elota, ubicado en el centro-sur del Estado de Sinaloa, es un ejemplo de la dinámica de las organizaciones migrantes. En la investigación realizada por Villegas (2015), se muestra que las organizaciones de migrantes elotenses se encuentran conformadas en tres organizaciones de oriundos: La Fraternidad Elotense de California (FEC), la Fraternidad Sinaloense La Cruz AC (FSLC) y el Club Paisanos Elotenses (CPE). Estas han potenciado las inversiones sociales a través del Programa 3x1. Asimismo, la organización que más se destaca es la FEC en California con el 46,56\% del total de las obras en el municipio, le sigue el CPE con el $39,52 \%$ y la FSLC con el $10 \%$.

El nivel de reconocimiento no es pleno en estas organizaciones, ya que son marcadas por el tipo de inversión o por la intención de convertirse en proyectos empresariales en su lugar de origen, $y$ no por el grado de representatividad y esfuerzos organizativos que llevaron a realizar sus inversiones. Lo interesante es que en este tipo de organizaciones, en la medida en que van sumando éxitos y compromisos, involucran a nuevos actores en el plano regional o internacional.

Dentro de estas organizaciones existen variantes entre los grupos de migrantes. Entre estas se encuentran sus formas de movilización $y$ gestión en las inversiones sociales, los diversos tipos de negociación que emprenden, la historicidad $y$ la presencia de los grupos de migrantes con diferentes intensidades en función de número de migrantes expulsados hacia Estados Unidos. Tales diferencias les permiten plantear una serie de acercamientos e involucrarse en las agendas de las políticas públicas de los lugares de origen; sin embargo, debido a las diferentes intensidades migratorias, unas con más migrantes y otras con menos, no todos responden de la misma manera. Por ello, existen distintas formas de integración de la organización, algunas promovidas por líderes locales o comunitarios, incluso gubernamentales, pero siempre bajo la bandera del otro, "del que se fue, pero quiere estar en la comunidad".

En esta confluencia de organizaciones del mismo municipio, la única organización 
que se encuentra registrada ante el Instituto de los Mexicanos en Exterior (IME) es el CPE, pero todas están inscritas en el Programa 3x1. Esta organización de oriundos fue conformada en el 2011, teniendo su base en la ciudad de Lynwood, California, dentro de la zona de Los Ángeles; como característica particular, en ese momento su presidenta era una mujer, algo poco común en este tipo de organizaciones. $\mathrm{Su}$ creación se debió al, en ese entonces, presidente municipal de Elota, Rodríguez Castillo, que con el apoyo familiar se organizaron para conformar una mesa directiva y así obtener la toma de nota, registrado como club oficial ante el IME, $y$ así poder acceder a los recursos más altos del Programa (Villegas, 2015).

Durante 2002-2013, fue el periodo más productivo; las acciones del CPE se reflejaron en 18 obras en el municipio, lo que equivale a $10,45 \%$ del total estatal. Estas tuvieron un valor de inversión de más de 20 millones de pesos, cantidad que constituyó $16,51 \%$ de todas las inversiones realizadas en Sinaloa a lo largo del periodo señalado. Al desagregar las aportaciones realizadas por cada agente, el gobierno municipal de Elota es el que más contribuciones monetarias realizó de todos los municipios sinaloenses (23,17\% del total). Le siguen las aportaciones realizadas por el gobierno del Estado con $17,75 \%$, después están las contribuciones de las organizaciones migrantes elotenses con $14,62 \%$ del total aportado por el resto de las organizaciones migrantes de Sinaloa; $y$ finalmente, están los fondos erogados por el Gobierno Federal que constituyen el 13,33\% de lo aportado por esta instancia hacia el resto de los municipios.

TABLA 2

OBRAS REALIZADAS CON EL PROGRAMA 3X1 EN SINALOA POR MUNICIPIO, 2002-2012

\begin{tabular}{c|ccccccccccc|c}
\hline MUNICIPIOS & 2012 & 2011 & 2010 & 2009 & 2008 & 2007 & 2006 & 2005 & 2004 & 2003 & 2002 & $\begin{array}{c}\text { TOTAL DE } \\
\text { OBRAS }\end{array}$ \\
\hline Ahome & 2 & 11 & 3 & - & 5 & - & - & - & - & 1 & 1 & 23 \\
Angostura & - & - & - & 1 & 1 & - & - & - & - & - & 1 & 3 \\
Badiraguato & - & - & - & - & 3 & - & - & - & - & - & - & 3 \\
Choix & 3 & 5 & 2 & 2 & 2 & 1 & - & - & - & - & - & 15 \\
Concordia & - & - & - & - & - & - & - & 1 & - & - & - & 1 \\
Cosalá & - & - & - & - & - & - & - & - & - & - & - & 0 \\
Culiacán & 4 & 5 & 1 & 1 & - & - & - & - & - & - & 6 & 17 \\
El Fuerte & 1 & - & - & - & - & - & 1 & 1 & - & 2 & 2 & 7 \\
Elota & 1 & 1 & 3 & 2 & 1 & 6 & 1 & 1 & - & 1 & 1 & 18 \\
Escuinapa & - & - & - & - & - & - & - & - & - & 2 & 1 & 3 \\
Guasave & 2 & 9 & 5 & 7 & 1 & 5 & 5 & - & - & 1 & 5 & 40 \\
Mazatlán & - & - & - & 1 & - & - & - & - & - & - & - & 1 \\
Mocorito & - & - & - & & 1 & - & - & 1 & - & - & 3 & 5 \\
Navolato & 1 & - & - & - & - & - & - & - & - & - & 2 & 3 \\
Rosario & - & - & - & - & - & 5 & 2 & 1 & - & - & - & 8 \\
S. Alvarado & - & - & - & - & - & - & - & - & - & 2 & 1 & 3 \\
San Ignacio & - & - & - & - & - & 2 & 2 & - & - & 4 & - & 8 \\
Sinaloa de L. & - & - & 6 & - & 2 & 1 & 1 & 1 & - & 5 & 1 & 17 \\
\hline TOTAL DE & 14 & 31 & 20 & 14 & 16 & 20 & 12 & 6 & 0 & 18 & 24 & 175 \\
OBRAS POR AÑO & 14 & & & & & & & & &
\end{tabular}

Fuente: Elaboración propia con datos de SEDESOL, 2014. 
La tabla 2 muestra que los municipios con menor número de obras construidas son Angostura, Badiraguato, Escuinapa, Navolato y Salvador Alvarado con 3 respectivamente, seguido de Mazatlán y Concordia con una sola obra, y Cosalá con ninguna obra realizada. Este último municipio posee el más alto nivel de pobreza y en situación extrema según el Consejo Nacional de Evaluación de la Política de Desarrollo Social (Coneval, 2012). Por lo tanto, esto ha dado como resultado que en el Estado de Sinaloa se construyeran en promedio 18 obras con una inversión de 5.75 millones de pesos anualmente, pero también refleja la vulnerabilidad de los diferentes acercamientos del Estado con los migrantes. Esta "política" de acercamiento con los migrantes se gesta mediante viajes $y$ visitas de las autoridades gubernamentales, principalmente en su nivel federal y estatal, con los líderes de las organizaciones de las diásporas locales en los principales lugares de concentración de migrantes en Estados Unidos (Valenzuela, 2007).

En estos encuentros se presentan una serie de señalamientos principalmente sobre la continuidad de los programas y la falta de transparencia, desconfianza por el uso de recursos monetarios por las obras programadas e incluso, en el abuso del ejercicio del poder para tomar decisiones por parte de las autoridades por encima de las organizaciones de migrantes (Yescas, 2018). Sin embargo, para algunos presidentes municipales de Sinaloa, estas acciones les permiten, además de "pilotear" o ensayar nuevas formas de tratar a la migración, tener un mejor desempeño en la infraestructura pública de sus comunidades. Ahora bien, aquí las organizaciones de migrantes ocupan el espacio público que no solo les otorgan las autoridades, sino el espacio público que se han apropiado por sus medios. Aquí es donde hay posibilidades de crear espacios transnacionales sólidos, algunos fuertemente politizados y que generan escenarios de negociación social entre migrantes y sus gobiernos. A partir de lo que postulan Guarnizo, Sánchez y Roach (2003), se sostiene que la intervención del Estado ha permitido recrear espacios transnacionales, y así los migrantes participan en el ámbito político en sus comunidades de origen e influyen en los procesos políticos y comunitarios.

De igual manera para Moctezuma (2011), "las prácticas y compromisos de los migrantes incluyen las identidades, las dimensiones políticas y culturales de la organización" (p. 94), donde históricamente se ha documentado que los clubes de migrantes mexicanos no necesitan al programa.

Así pues, la FEC permitió que se construyera una secundaria técnica, electrificación y alcantarillado en el municipio y además se gestionaron recursos para el Programa "Cruzada Nacional contra el hambre" en el 2014, gracias al programa 3x1 (El Debate, marzo 2014). Lo más destacable es la cuantiosa y constante participación del gobierno municipal y de los clubes elotenses en el desarrollo del Programa 3x1, ya que supera, en términos absolutos, a municipios de Ahome, Guasave o Culiacán. Estas entidades rebasan por mucho a Elota en la cantidad de emigrantes y la cantidad de remesas recibidas, presupuesto público y extensión territorial, pero las distintas organizaciones de migrantes elotenses superan en términos relativos al resto de las organizaciones migrantes sinaloenses participes en el Programa 3x1.

Para Villegas (2015) "a nivel municipal gobierno de Elota es el que mayores inversiones ha realizado ya que aportó 6 millones de pesos mexicanos, lo que equivale a 30.37 $\%$ respecto al total de las contribuciones al Programa en el municipio" (p.160); le siguen las inversiones federales con el $27,26 \%$ del total municipal. Con estas cifras se da cuenta de que, aunque el gobierno estatal apoyó en mayor medida a Elota en comparación con los otros municipios sinaloenses, sus contribuciones no sobrepasan a lo aportado por el resto de los agentes a nivel municipal.

Para el caso de las organizaciones de Elota, no se cumple aquella noción de que a nivel estatal existe una una nula política institucional entre el Gobierno y comunidades de emigrados. Como menciona Valenzuela (2007), lo que se observa por un lado es una relación "cómoda, orgánica, o funcional", mientras que por el otro, "superficial e intrascendente". Entonces, la relación entre los 
migrantes organizados y el gobierno de Sinaloa es de doble cara; por un lado, se reconoce la fuerza de la organización de migrantes a través de las inversiones sociales, pero por el otro, el gobierno estatal sabe de las bondades de las remesas en general $y$ el impacto familiar $y$ local en las comunidades.

\section{GESTIONES TRANSNACIONALES Y DESARROLLO LOCAL EN SINALOA}

Para los principales municipios expulsores de migrantes en Sinaloa, el diseño del programa $3 \mathrm{x} 1$ tienen como sostén y respaldo a las propias organizaciones de migrantes, principalmente a las que ya están organizadas y no han buscado o intensificado el esfuerzo en la promoción de organizarse y de tener adhesión a su matría. En sí, la participación de las autoridades locales $y$ los clubes de migrantes en el programa 3x1 no es autónoma en su totalidad. Estas se ajustan principalmente a las reglas de operación del programa y a las diversas formas de negociar su ejecución. Sin embargo, en la práctica son varios los casos en que los municipios negocian con los clubes de oriundos, el apoyo de una obra a cambio de conceder obras más allá de los intereses originales de los clubes de migrantes (cuadro 2).

CUADRO 2

BENEFICIOS Y PROBLEMÁTICA DEL PROGRAMA 3X1

\begin{tabular}{ll}
\hline \multicolumn{1}{c}{ BENEFICIOS } & \multicolumn{1}{c}{ PROBLEMÁTICA } \\
\hline Su enfoque es de filantropía social. & $\begin{array}{l}\text { Su base son las remesas colectivas por lo que dependen } \\
\text { de la organización y de los fondos de los migrantes. } \\
\text { La responsabilidad de la ejecución del proyecto queda en } \\
\text { Responde a motivaciones humanitarias y de prestigio } \\
\text { social (1). }\end{array}$ \\
$\begin{array}{l}\text { Pueden crear proyectos empresariales. } \\
\text { Aprobada la obra, los clubes y beneficiarios locales no }\end{array}$ \\
$\begin{array}{ll}\text { El Migrante y la sociedad civil son activos promotores de } \\
\text { los programas. }\end{array}$ & $\begin{array}{l}\text { La capacidad de promoción y captación de fondos de } \\
\text { los clubes ha rebasado la capacidad presupuestal de los } \\
\text { gobiernos locales (1). }\end{array}$ \\
$\begin{array}{l}\text { Buena aceptación entre comunidades y entre los } \\
\text { migrantes (1). }\end{array}$ & $\begin{array}{l}\text { No se hacen previsiones para su mantenimiento } \\
\text { posterior. }\end{array}$ \\
$\begin{array}{l}\text { Sirven para recrear y fomentar las acciones sociales de } \\
\text { una sociedad. }\end{array}$ & Exceso de burocratismo. \\
\hline
\end{tabular}

Fuente: Pintor, 2011.

Nota 1: García Zamora, 2003, p. 19-51.

La problemática central en la gestión $y$ negociación es la toma de decisiones para las obras a construir. Aquí se pone a prueba el grado de autonomía y el interés gubernamental para la puesta en marcha de obras que beneficien a las comunidades. Como señalan Pintor (2015) y Valenzuela (2007) al coincidir sobre el caso de comunidades migrantes sinaloenses, las cuales el apoyo se gesta a partir de la negociación y convencimiento a las autoridades gubernamentales.
Si bien es importante la figura del Estado en la movilización y activación de los componentes de la sociedad —ya sea emigrada o nopuede permitir esquemas democratizadores mucho más incluyentes, donde ambos puedan ayudar a crear nuevas y más amplias agendas de gobierno. Por ejemplo, Escribá, Meseguer $y$ Wright (2013) encuentran que las remesas hacen a los votantes menos dependientes de las redes del clientelismo político, e incrementan la posibilidad de la democratización, ya que estas personas no se encuentran asociadas con la 
corrupción del Estado y del soporte electoral de los partidos dominantes.

También se observa el impacto social y político en pro de la democracia, como menciona Wadell (2014) "a través de las remesas colectivas en lugares donde se invierte, estás hacen posible que las personas se involucren más en las agendas gubernamentales locales y suceda alternancia" (p.116). En este sentido, gobernar con instrumentos de políticas públicas no se entiende como producto de decisiones unidireccionales, sino como producto de una larga serie de decisiones, programas, intenciones e interacciones organizacionales que afectan la vida de la sociedad. En este sentido, gobernar con instrumentos de políticas públicas no se entiende como producto de decisiones unidireccionales, sino como producto de una larga serie de decisiones, programas, intenciones e interacciones organizacionales que afectan la vida de la sociedad.

De igual forma, la participación de los clubes de migrantes, en conjunción con los no migrantes debe de reflejarse en la fiscalización de las obras públicas, proyectos en común en urbanización, electrificación, conservación del patrimonio histórico local (Fung y Olin, 2001), atención y asesoría legal migrante o inclusión política de los migrantes. Estas acciones conjuntas entre las organizaciones de los migrantes y los presidentes municipales, los asuntos de obra pública y su decisión sobre financiamiento, podrían resolverse en el principio de participación ciudadana transnacional. Bajo este principio, se le otorga reconocimiento a los migrantes organizados, no solo a nivel federal, donde se encuentra el programa, sino también en el municipio, donde incluso pueden pasar a coparticipar con el gobierno local y negociar con nuevos actores como las empresas, organismos federales, internacionales y fundaciones.

Con base en lo anterior, la membresía que ejercerá el migrante desde el extranjero, permitirá dar significado a su propio entorno por ser "objetivamente" parte de esa estructura llamada comunidad, ahora convertida en transnacional ${ }^{5}$. De igual forma, se refuerzan o

$5 \quad$ Es inseparable de estas acciones las conexiones que establecen la dinámica migratoria, por establecen una serie de lealtades y compromisos que defienden y comparte con quienes no han emigrado, pero que son parte de la misma comunidad. Por lo tanto, el funcionamiento de las organizaciones, no solo se centra en el migrante sino en los propios "espacios sociales transnacionales" que van creando. Estos espacios constituyen una importante estructura de referencia para los posicionamientos sociales que determinan la praxis de la vida cotidiana, las identidades y los proyectos biográficos (laborales) y que, simultáneamente, trascienden el contexto social de las sociedades nacionales (Pries, 2000).

Entonces, efectivamente crea un nuevo institucionalismo del transnacionalismo y que permite conformar una extensa gama de procesos organizativos, que van desde formas de representación permeadas por lo religioso, simbólico, local o comunitario, hasta la conformación de verdaderos frentes o espacios que influyen en el diseño de las políticas públicas locales (Valenzuela y Lanly, 2004).

\section{CONCLUSIONES}

El diseño de políticas públicas de inclusión entre la sociedad emigrada y sus gobiernos es un tema que cada vez tomará más pertinencia, dada la nueva era y masividad organizada en la migración mexicana a Estados Unidos. Las formas de representación y organización de los migrantes han permitido cambiar la agenda pública en cuanto a la implementación de programas sociales en comunidades de migrantes.

Si bien es cierto que la participación del Estado es importante para la regulación, es necesario plantear que las organizaciones de migrantes mexicanos han creado diferentes programas, que por su naturaleza, se convierten en auténticos proyectos de iniciativas públicas que tratan de incentivar el desarrollo de sus comunidades de origen; como por ejemplo, el caso de la Fraternidad Sinaloense en California (FSC).

ejemplo, a través de los polleros (traficantes de migrantes), comercio informal y organizaciones no gubernamentales que defiende al migrante (Molina $y$ Vedia, 2014). 
Haciendo un balance, el club de migrantes de Agua Verde y la Fraternidad Elotense en California ejecutan acciones en nombre del bienestar de su comunidad de origen, la adhesión y su membresía activa comunitaria, los lleva a no desligarse de las acciones sociales de índole cultural, cívica y política, sujetas a prácticas y vínculos transnacionales.

Este tipo de asociaciones son estructuras organizativas complejas y permiten que sus relaciones estén sustentadas en los liderazgos de las organizaciones y potencialicen sus destrezas de negociación en el plano binacional, sobre el cual se mueven y donde sería imposible pensar en instaurar, ya no inversiones sociales, sino programas públicos de largo alcance. Se puede sostener que existe un empoderamiento del migrante organizado, que a lo largo de décadas de negociación y gestión realizan actividades cívicas y filantrópicas mediante relaciones comunitarias y de solidaridad.

Sin embargo, este empoderamiento es compartido, como lo muestra el caso de Elota, en el cual su estructura y fortaleza es promovida en conjunto con el liderazgo y la coparticipación de los presidentes municipales, diseñando e implementando políticas públicas de corte local junto con la reconstrucción transnacional comunitaria.

No se trata de asumir una postura en la cual los clubes de migrantes son la panacea ante los problemas de índole estructural en las comunidades de origen, ya sea de manera autónoma o con la ayuda de sus gobiernos de origen. Más bien, se debe prestar atención a la naturaleza $y$ desarrollo que han logrado estas organizaciones de migrantes, a partir del envío de remesas y su reconocimento como factor de desarrollo. También es necesario que los clubes de migrantes se coordinen con los gobiernos, y más allá de contribuir al desarrollo regional, se implemente una cooperación binacional o transnacional en la cual se reconozca la ciudadanía extendida y sus derechos.

\section{REFERENCIAS}

Alba, F. (2010). Extendiendo el alcance de las políticas públicas en materia migratoria. En P. Leite y S. E. Giorguli (Coord.),
Reflexiones a la emigración mexicana (pp.17-26). México D.F.: CONAPO.

Alarcón, R. (2004). Las remesas colectivas y las asociaciones de migrantes mexicanos en los Estados Unidos. En Germán A. Z. H. (Coord.), Remesas de los mexicanos y centroamericanos en Estados Unidos (pp. 159-184). México D.F.: M.A. Porrúa.

Barnes, J. (1954). Class and communities in a Norwegian island parish. Human Relations, 7 (1), 39-58.

Consejo Nacional de Evaluación de la Política de Desarrollo Social. (2012). Informe de pobreza y evaluación en el estado de Sinaloa. Consejo Nacional de Evaluación de la Politica de Desarrollo Social. Recuperado de http://www.coneval.org. $\mathrm{mx} /$ coordinacion/entidades/Documents/ Sinaloa/principal/25informe2012.pdf

Elota ingresa a la Cruzada Nacional contra el hambre. (13/03/2014). El Debate de Culiacán, p.12.

Enríquez, R. (2000). Redes sociales y pobreza, mitos y realidades. Revista de Estudios de Género. La Ventana, (11), 36-72.

Escala, L. (2014). Asociaciones de inmigrantes mexicanos en Estados Unidos: logros y desafíos en tiempos recientes. Desacatos, (46), 52-69.

Escribá, F., Meseguer, M., Wright, J. (2013). Remittances and Democration. International Studies Quarterly, 3 (59), 571-586.

Fitzgerald, D. (2014). Nación de emigrantes: Cómo maneja México su migración. Tijuana: Colef.

Fung, A. y Wright E. (2001). Entorno al gobierno participativo con poder de decisión. En Canto, M., Participación ciudadana en las políticas públicas (150-175). México. D.F.: Siglo XxI.

García, R. (octubre-diciembre de 2003). Los proyectos productivos con los emigrantes en México, hoy, Arenas, 5.

García, R. (2005). Comunidades transnacionales México-Estados Unidos. Circuito Zacatecas-Estados Unidos. Estudios del Desarrollo de la Universidad Autónoma de Zacatecas, Zacatecas. Recuperado de 
http://www.estudiosdeldesarrollo.mx/ administracion/documentos/circuito_ zacatecas.pdf

Gibson, J., Ivancevich J. y Donnelly, J. (1994). Las organizaciones, conducta, proceso. Wilmington: Addison-Wesley Iberoamericana.

Giménez, G. (2010). Cultura, identidad y procesos de individualización. México D.F.: Universidad Autónoma de MéxicoInstituto de Investigaciones Sociales. Recuperado de http://perio.unlp.edu.ar/ teorias2/textos/articulos/gimenez.pdf

Goldring, L. (2002). The Mexican State and Transmigrant Organizations: Negotiating the Boundaries of Membership and Participation. Latin American Research Review, 37 (3), 55-99.

Goldring, L. (2005). Implicaciones sociales $y$ políticas de las remesas familiares $y$ colectivas. En R. Delgado y B. Knerr. (Coord.), Contribuciones al análisis de la migración internacional y el desarrollo regional en México (pp.67-94). México D.F.: M.A. Porrúa.

González, C. (2006). Del acercamiento a la inclusión institucional: la experiencia del Instituto de los Mexicanos en el Exterior. En C. González, Relaciones Estado Diáspora: aproximaciones desde cuatro continentes, (pp.181-220). México : мA. Porrúa-SER.

Guarnizo, L., Sánchez, A. I. y Roach, E. (2003). Desconfianza, solidaridad fragmentada y migración transnacional: los colombianos en la ciudad de Nueva York y Los Ángeles. En L. Guarnizo, A. Portes y P. Landolt (Coord.), La Globalización desde abajo: transnacionalismo inmigrante $y$ desarrollo. La experiencias de Estados Unidos y América Latina (279-305). México D.F.: M.A. Porrúa y FLACSO.

Hernández, M. (mayo de 2007). Participación ciudadana y el rescate de la ciudad. Revista Invi, 59 (22), 13- 34.

Lanly, G. y Valenzuela, B. (2004). La política trasnacional de la nueva sociedad migrante. En G. Lanly y B. Valenzuela. Clubes de migrantes oriundos mexicanos en los
Estados Unidos (pp.11-36). Guadalajara, México: Universidad de Guadalajara.

Moctezuma, M. (enero-abril, 2008). El migrante colectivo transnacional: Senda que avanza y reflexión que se estanca. Sociológicas, 23(60), 93-111.

Moctezuma, M. (2011). La transnacionalidad de los sujetos. Dimensiones, metodologías y prácticas convergentes de los migrantes mexicanos en Estados Unidos. México D.F.: M.A. Porrúa y Universidad Autónoma de Zacatecas.

Molina y Vedia, S. (2014). ¿Qué es la transmigración? En S. Molina; V. del Castillo y A. Méndez, Migración, organizaciones civiles y transmigración. Redes e interdependencia (17-41). México D.F.: UNAM -IIE.

Morán, L. (2002). Las organizaciones de Migrantes, su impacto y evolución en la recepción de personas y el envío de recursos. Seminario de Nuevas Tendencias y Desafíos de la Migración Internacional México-Estados Unidos, celebrado en noviembre de 2002. México D.F.

Padilla, J. M. (2000). Emigración internacional y remesas de Zacatecas. Comercio exterior, 50 (5), 363-370.

Pintor, R., y García, I. (2008). Inversiones de los Clubes de migrantes Sinaloenses en Estados Unidos, realizadas en sus lugares de origen. Revista Sinaloa: Causa Común, (34), 9-13.

Pintor, R. (2011). El habitus y los campos transnacionales en el proceso del transnacionalismo migrante. Migraciones Internacionales, 6 (2), 159-192.

Pintor, R. (2015). El otro Agua Verde, Sinaloa. Procesos Trasnacionales migrantes. Culican, Sinaloa: UAS.

Pries, L. (2000). Una nueva cara de la migración globalizada: el surgimiento de nuevos espacios sociales transnacionales y plurilocales. Trabajo, 2, (3), 363-370.

Revista de la Fraternidad Sinaloense en California. (1999). Informe de labores. Pasadena, California.

Revista de la Fraternidad Sinaloense en California. (2000). Informe de labores. Pasadena, California. 
Revista de la Fraternidad Sinaloense en California. (2008). Informe de labores. Pasadena, California, Edición especial.

Rouse, R. (1992). Making sense of settlement: Class transformation, cultural struggle, and transnationalism among Mexican migrants in the United States. Annals of the New York Academy of Sciences, 645, 25-52. Doi https://doi. org/10.1111/j.1749-6632.1992.tb33485.x

Sánchez S., Garcia, I. y Pintor, R. (2015). Revisión crítica de los programas de coinversión entre comunidades migrantes transnacionales y los gobiernos federal y locales en México. Identidades, 9 (5), 25-42.

Secretaria de Desarrollo Social (SEDESOL) (2017). Programa 3x1 para migrantes. Recuperado de https://www.gob.mx/.../elprograma-3-x-1-para-migrantes-ha-beneficiado-a-mas-de-14-millones ?idiom=es

Smith, C. (2006). México en Nueva York: Vidas transnacionales de los migrantes mexicanos entre Puebla y Nueva York. D.F. México: M. Á. Porrúa-UAZ.

Torres, F. (2001). Remittances for Small-Scale Infrastructure and Small Enterprise Development in Mexico. Evidence from the Public-Private Infrastructure Advisory Facility Study. Approaches to Increasing the Productive Value of Remittances. Washington, D.C: IAF, ECLAC and World Bank Conference.

Valenzuela, B. (2007). El papel de las organizaciones de migrantes en la definición de acciones y políticas públicas estatales hacia la migración a los Estados Unidos. En R. Fernández de Castro, Politicas
Migratorias en los Estados de México (pp.17-43). D.F, México: M. A. Porrúa.

Valenzuela, B. y Lanly, G. (2004). El papel de las organizaciones y los gobiernos mexicanos. En G. Lanly y B.Valenzuela (Comps.), Clubes de migrantes oriundos en los Estados Unidos, (30-57). Guadalajara, México: Universidad de Guadalajara.

Villegas , F.E. (2015). El impacto de las remesas colectivas en el desarrollo comunitario. Estudio de caso del municipio de Elota, Sinaloa 2002-2013 (Tesis de maestría), Universidad Autónoma de Sinaloa, Mazatlán.

Waddell, B. (2014). Remitting democracy? The role of migrant remittances in promoting social and political change in Guanajuato, México. Journal of Community Positive Practices, 1(14), 116-130.

Yescas, Alvarado, C. (2018). Irregularidades de parte de instituciones en programa 3x1. Recuperado de http://enlaceconexionentreculturas.com/irregularidades-de-parte-de-instituciones-en-programa-3x1-denuncia-alvaviola-garcia.

Zabin C. y Escala L. (2002). From Civic Association to Political Participation: Mexican Hometowns Association and Mexican Immigrant Political Environment in Los Angeles. Frontera Norte, (27), 7- 42.

Fecha de ingreso: 09/10/2018 Fecha de aprobación: 23/07/2019 